\title{
Classical and Quantum Wormholes in Einstein-Yang-Mills Theory
}

\author{
Hongsu Kim \\ Department of Physics \\ Ewha Women's University, Seoul 120-750, KOREA
}

(May, 1997)

\begin{abstract}
Wormhole spacetimes may be responsible for the possible loss of quantum coherence and the introduction of additional fundamental quantum indeterminancy of the values of constants of nature. As a system which is known to admit such classical wormhole solutions, Einstein-Yang-Mills (EYM) theory is revisited. Since the classical wormhole instanton solution in this theory has been studied extensively thus far, in the present work, "quantum wormholes" are explored. Namely in the context of a minisuperspace quantum cosmology model based on this EYM theory, "quantum wormhole", defined as a state represented by a solution to the Wheeler-DeWitt equation satisfying an appropriate wormhole boundary condition, is discussed. Finally, it is proposed that the minisuperspace model based on this theory in the presence of the cosmological constant may serve as a simple yet interesting system displaying an overall picture of entire universe's history from the deep quantum domain all the way to the classical domain.
\end{abstract}

PACS numbers : $04.20,98.80 . \mathrm{H}, 11.10 . \mathrm{C}$

Key words : Wormhole, Einstein-Yang-Mills theory

Typeset using REVTEX 


\section{Introduction}

If one identifies the spacetime metric as the relevant gravitational field subject to the quantization, the topology of spacetime is expected to fluctuate on scales of the order of the Planck length $l_{p}=M_{p}^{-1}$. And of all types of conceivable spacetime fluctuations, major concern has been the "wormhole configuration" which is an object that can be loosely defined as the instanton which is a saddle point of the Euclidean action making dominant contribution to the topology changing transition amplitude. Then one of the most crucial effects the wormhole (or more generally, these spacetime fluctuations) may have on low-energy physics could be the possible effective loss of quantum coherence [1-3]. For example, one may speculate the situation where "baby universes" are pinched off and carry away information. Then this kind of stereotypical information loss can lead to an effective loss of quantum coherence as viewed by the macroscopic observer who cannot measure the quantum state of the baby universes. This issue of the possible loss of quantum coherence in (mostly the semiclassical approximations of) quantum gravity stood at the center of debates some time ago [1-3]. In a more careful and concrete analysis, however, these arguments concerning the possible quantum incoherence should be taken with some caution. For example, there is an argument by Coleman [3] that in the context of "many universe" interpretation (i.e., the third quantization formalism), the quantum incoherence will not be observed, namely the quantum coherence will be restored. In fact, of all the possible effects of the fluctuations in spacetime topology on the low energy physics, the most provocative one that immediately attracted enormous excitement was the advocation initiated by Baum [1] and by Hawking [1] and then refined later by Coleman [3] that the wormholes have ultimately an effect of turning all the constants of nature into "dynamical random variables". Thus this Baum-Hawking-Coleman (BHC) mechanism leads to a striking conclusion that the effects of (particularly) wormholes introduces into the low energy physics a fundamental quantum indeterminancy of the values of the constants of nature which can be thought of as an additional degree of uncertainty over the usual uncertainty in quantum mechanics. Unlike the issue of the loss of quantum coherence discussed earlier, however, this BHC-mechanism does not simply imply the elimi- 
nation of the classical predictability of nature by the effect of quantum gravity. For instance, the BHC-mechanism actually leads to the prediction that the most probable value of the fully-renormalized cosmological constant is zero, indeed in exact agreement with the observation. For the detailed arguments involved in the BHC-mechanism particularly concerning the most probable value of the fully-renormalized cosmological constant, we refer the reader to the literature $[1,3]$. But it seems fair to mention that the formulation of wormhole physics particularly the one put forward by Coleman is not without some inherent flaws. First, logically the Coleman's wormhole physics formulation had faced some severe criticisms such as "sliding of Newton's constant problem" [7] and "large wormhole catastrophe" [8] which seem to be unfortunately quite generic. However, it seems that the most fundamental and crucial difficulty associated with Coleman's formulation is the use of saddle point approximation to the Euclidean path integral for quantum gravity since, as is well-known, the Euclidean Einstein-Hilbert action is not bounded below [9].

Now, then, note that both issues discussed thus far, namely the loss of quantum coherence and the determination of probability distribution for constants of nature (which are now random variables), are clearly based on the assumption that there are wormhole instantons as saddle points of the Euclidean action of the theory under consideration. Therefore unless one can demonstrate that there are large class of theories comprised of gravity with or without matter which admit Planck-sized wormhole instantons as solutions to the classical field equations, the discussion above on interesting effects of wormholes on low energy physics will lose much of its meaning. Unfortunately, thus far only a handful of restricted classes of theories are known to possess classical wormhole instanton solutions and they include ; Einstein-Kalb-Ramond (KR) antisymmetric tensor theory [4], Einstein-Yang- Mills theory [5] and Einstein-complex scalar field theory in the presence of spontaneous symmetry breaking [6]. The classical wormhole instanton solutions and the semiclassical analysis of their effects on low energy physics in these theories had been thoroughly studied in the literature. Here in the present work, we revisit the Einstein-Yang-Mills (EYM) theory of Hosoya and Ogura [5] which is known to admit classical, Euclidean wormhole instanton solutions. One 
may wonder why anybody should repeatedly go through a well-studied theory like this one. Although the "classical" wormhole instanton as a solution to the classical field equations and its effects on low energy physics had been studied extensively, almost no attempt has been made concerning the serious study of "quantum" wormholes in the same theory. Thus in the present work we attempt to explore the existence of quantum wormholes in EYM theory. Now we describe briefly the approach we shall employ to explore the physics of quantum wormholes in our theory. And to do so, it seems necessary to distinguish between the definition of "classical" wormhole and that of "quantum" wormhole. In the classical sense, wormholes are Euclidean metrics which are solutions to the Euclidean classical field equations representing spacetimes consisting of two asymptotically Euclidean regions joined by a narrow tube or throat. In the quantum regime, on the other hand, and particularly in the context of the canonical quantum cosmology, quantum wormholes may be identified with a state or an excitation represented by a solution to the Wheeler-DeWitt equation satisfying a certain boundary condition describing the wormhole configuration. An widely-accepted such "wormhole boundary condition" is the one advocated by Hawking and Page [10]. And it states that wormhole wave functions are supposed to behave in such a way that they are damped, say, exponentially for large 3-geometries $(\sqrt{h} \rightarrow \infty)$ and are regular in some suitable way when the 3 -geometry collapses to zero $(\sqrt{h} \rightarrow 0)$. Thus we shall construct a minisuperspace quantum cosmology model possessing SO(4)-symmetry based on the EYM theory and attempt to solve associated Wheeler-DeWitt equation. It will be demonstrated that there indeed is a solution to the Wheeler-DeWitt equation whose asymptotic behaviors satisfy the "wormhole boundary condition" stated above and hence can be identified with a wormhole wave function, namely a universe wave function for quantum wormholes.

This paper is organized as follows : In sect.II, we recapitulate classical wormhole instanton solutions and the semiclassical analysis of their effects on low energy physics in EYM theory. Sect.III will be devoted to the study of quantum wormholes in this theory employing the approach described above. Finally in sect.IV, we summarize the results of our study and discuss their physical implications. 


\section{Classical wormhole physics in EYM theory}

We begin by reviewing the classical wormhole solution in EYM theory and its implications on low energy physics in a rather different context from the one in the literature [5]. Consider a system consisting of Yang-Mills (YM) theory of SU(2) gauge field coupled to Einstein gravity in the presence of the cosmological constant. This Einstein-Yang-Mills theory is represented by the Euclidean action [5]

$$
I_{E Y M}=\int_{M} d^{4} x \sqrt{g}\left[\Lambda-\frac{M_{p}^{2}}{16 \pi} R+\frac{1}{4 g_{c}^{2}} F_{\mu \nu}^{a} F^{a \mu \nu}\right]-\int_{\partial M} d^{3} x \sqrt{h} \frac{M_{p}^{2}}{8 \pi}\left(K-K_{0}\right)
$$

where $F_{\mu \nu}^{a}$ is the field strength of the YM gauge field $A_{\mu}^{a}$ with $a=1,2,3$ being the $\mathrm{SU}(2)$ group index and $g_{c}$ being the gauge coupling constant. We also added Gibbons-Hawking gravitational boundary term on $\partial M$ with $h$ being the metric induced on $\partial M$ and $K$ being the trace of the second fundamental form of $\partial M$.

Then by extremizing the action above with respect to the metric $g_{\mu \nu}$ and the YM gauge field $A_{\mu}^{a}$, one gets the following classical field equations respectively

$$
\begin{array}{ll} 
& R_{\mu \nu}-\frac{1}{2} g_{\mu \nu} R+\frac{8 \pi}{M_{p}^{2}} \Lambda g_{\mu \nu}=\frac{8 \pi}{M_{p}^{2}} T_{\mu \nu} \\
\text { with } \quad & T_{\mu \nu}=\frac{1}{g_{c}^{2}}\left[F_{\mu \alpha}^{a} F_{\nu}^{a \alpha}-\frac{1}{4} g_{\mu \nu}\left(F_{\alpha \beta}^{a} F^{\alpha \beta}\right)\right], \\
& D_{\mu}\left[\sqrt{g} F^{a \mu \nu}\right]=0
\end{array}
$$

where and henceforth we use the notations $F_{\mu \nu}^{a}=\partial_{\mu} A_{\nu}^{a}-\partial_{\nu} A_{\mu}^{a}+\epsilon^{a b c} A_{\mu}^{b} A_{\nu}^{c}, D_{\mu}^{a c}=\partial_{\mu} \delta^{a c}+$ $\epsilon^{a b c} A_{\mu}^{b}, A_{\mu}=A_{\mu}^{a}\left(-i T^{a}\right)$ and $F_{\mu \nu}=F_{\mu \nu}^{a}\left(-i T^{a}\right)$ with $D_{\mu}^{a c}$ being the usual gauge-covariant derivative and $T^{a}$ 's are $\mathrm{SU}(2)$ group generators obeying the $\mathrm{SU}(2)$ Lie algebra $\left[T^{a}, T^{b}\right]=$ $i \epsilon^{a b c} T^{c}$ and the normalization $\operatorname{Tr}\left(T^{a} T^{b}\right)=\delta^{a b} / 2$.

Now we look for a Euclidean, $\mathrm{SO}(4)$-symmetric wormhole solution which is an instanton describing the nucleation of a, presumably, Planck-sized baby (spatially-closed ; $k=+1$ ) FRW-universe. To this end, we begin by taking $\mathrm{SO}(4)$-symmetric ansätz for the Euclidean metric and YM gauge field. First, the spatially-closed FRW-metric, which has the $\mathrm{SO}(4)$ symmetry, is given by 


$$
\begin{aligned}
d s^{2} & =g_{\mu \nu} d x^{\mu} d x^{\nu}=\delta_{A B} e^{A} \otimes e^{B} \\
& =\sigma^{2}\left[N^{2}(\tau) d \tau^{2}+a^{2}(\tau) \sigma^{a} \otimes \sigma^{a}\right]
\end{aligned}
$$

where $N(\tau)$ and $a(\tau)$ are lapse function and scale factor respectively and $\sigma^{2}=\left(2 / 3 \pi M_{p}^{2}\right)$ has been introduced for convenience. Throughout this work, we shall work with non-coordinate basis with indices $A, B=0, a(a=1,2,3)$ rather than with coordinate basis with indices $\mu, \nu=\tau, \theta, \phi, \psi$ where $(\theta, \phi, \psi)$ are Euler angles parametrizing the spatial section of the manifold which has the geometry of $S^{3}$. Then the non-coordinate basis 1 -forms can be read off as

$$
e^{A}=\left\{e^{0}=\sigma N(\tau) d \tau, \quad e^{a}=\sigma a(\tau) \sigma^{a}\right\}
$$

where $\left\{\sigma^{a}\right\}(a=1,2,3)$ form a basis on the 3 -sphere $S^{3}$ as just mentioned satisfying the $\mathrm{SU}(2)$ "Maurer-Cartan" structure equation

$$
d \sigma^{a}=\frac{1}{2} \epsilon^{a b c} \sigma^{b} \wedge \sigma^{c}
$$

and can be represented in terms of 3 -Euler angles $0 \leq \theta \leq \pi, 0 \leq \phi \leq 2 \pi$ and $0 \leq \psi \leq 4 \pi$, parametrizing $S^{3}$

$$
\begin{aligned}
\sigma^{1} & =\cos \psi d \theta+\sin \psi \sin \theta d \phi, \\
\sigma^{2} & =\sin \psi d \theta-\cos \psi \sin \theta d \phi, \\
\sigma^{3} & =d \psi+\cos \theta d \phi .
\end{aligned}
$$

For later use, we also write down the associated vierbein and its inverse using the definition, $e^{A}=e_{\mu}^{A} d x^{\mu}, e_{\mu}^{A} e_{B}^{\mu}=\delta_{B}^{A}$ and $e_{A}^{\mu} e_{\nu}^{A}=\delta_{\nu}^{\mu}$ where $x^{\mu}=(\tau, \theta, \phi, \psi)$.

$$
e_{\mu}^{A}=\sigma\left(\begin{array}{cccc}
N & 0 & 0 & 0 \\
0 & a \cos \psi & a \sin \psi \sin \theta & 0 \\
0 & a \sin \psi & -a \cos \psi \sin \theta & 0 \\
0 & 0 & a \cos \theta & a
\end{array}\right) \quad, \quad e_{A}^{\mu}=\frac{1}{\sigma}\left(\begin{array}{cccc}
\frac{1}{N} & 0 & 0 & 0 \\
0 & \frac{1}{a} \cos \psi & \frac{1}{a} \sin \psi & 0 \\
0 & \frac{\sin \psi}{a \sin \theta} & \frac{-\cos \psi}{a \sin \theta} & 0 \\
0 & \frac{-\sin \psi \cos \theta}{a \sin \theta} & \frac{\cos \psi \cos \theta}{a \sin \theta} & \frac{1}{a}
\end{array}\right) \text {. }
$$

Next we turn to the choice of $\mathrm{SO}(4)$-symmetric ansätz for the YM gauge field and its field strength. Note that the $\mathrm{SU}(2)$ group manifold is also $S^{3}$ just like it is the case for 
the geometry of the spatial section of the spacetime manifold. Thus one may choose the left-invariant 1-form $\left\{\sigma^{a}\right\}$ as the "common" basis for both group manifold and the spatial section of the spacetime manifold. And this indicates that there is now "mixing" between the group index in the YM field and the non-coordinate basis frame index since we choose to work with non-coordinate basis. In the present work, we restrict our interest only to the magnetically-charged solution, i.e., $F_{\tau i}^{a}=0(i=\theta, \phi, \psi)$. The motivation for this restriction will become clearer later on in the discussion of quantum wormhole physics. Namely from $F_{\mu \nu}^{a}=\left\{F_{\tau i}^{a}=0, F_{i j}^{a}\right\}(i, j=\theta, \phi, \psi)$, we can write down the $\mathrm{SO}(4)$-symmetric ansätz for YM field strength in non-coordinate basis indices

$$
\begin{aligned}
& F_{0 b}^{a}=e_{0}^{\mu} e_{b}^{\nu} F_{\mu \nu}^{a}=e_{0}^{\tau} e_{b}^{i} F_{\tau i}^{a}=0, \\
& F_{b c}^{a}=e_{b}^{\mu} e_{c}^{\nu} F_{\mu \nu}^{a}=e_{b}^{i} e_{c}^{j} F_{i j}^{a}=f(\tau) \epsilon^{a b c} .
\end{aligned}
$$

Note that the choice of the ansätz for $F_{b c}^{a}$ above is motivated by the choice of ansätz for the Kalb-Ramond antisymmetric tensor field strength, $H_{\mu \nu \lambda}=h(\tau) \epsilon_{\mu \nu \lambda}$ in the work by Giddings and Strominger [4] who looked for $\mathrm{SO}(4)$-symmetric wormhole instanton solutions as well. As a matter of fact, this is an essential common structure shared by the Einstein-antisymmetric tensor theory considered by Giddings and Strominger [4] and the present EYM theory first studied by Hosoya and Ogura [5] that allows the existence of classical wormhole solution. Also note that this kind of starting point of ours is different from that in the original work of Hosoya and Ogura [5] in which they begin by taking the $\mathrm{SO}(4)$-symmetric ansätz for the YM gauge potential rather than its field strength as we are doing here. The motivation for taking the ansätz for the field strength $F_{A B}^{a}$ will become clearer shortly.

Now, we would like to determine the condition that the function $f(\tau)$ appearing in $F_{b c}^{a}$ should meet and the form of the associated YM gauge potential $A_{B}^{a}$ by demanding only that they be consistent with the definition $F^{a}=d A^{a}+\frac{1}{2} \epsilon^{a b c} A^{b} \wedge A^{c}$ and obey the Bianchi identity $D F^{a}=d F^{a}-\epsilon^{a b c} F^{b} \wedge A^{c}=0$. And to do so, we choose to take the temporal gauge $A_{\tau}^{a}=0$ (or equivalently $A_{0}^{a}=0$ in non-coordinate basis). This gauge choice is indeed natural since the spacetime is homogeneous and isotropic thus depends only on $\tau$-coordinate, there 
is no gauge freedom associated with the space-dependent gauge transformation. Now we begin with the definition of the YM field strength $F^{a}=d A^{a}+\frac{1}{2} \epsilon^{a b c} A^{b} \wedge A^{c}$. On the one hand, using the YM gauge potential expressed in non-coordinate basis (in $A_{\tau}^{a}=0$ gauge) $A^{a}=A_{\mu}^{a} d x^{\mu}=A_{b}^{a} e^{b}(b=1,2,3)$, one can evaluate the above defining equation for the field strength with the help of Maurer-Cartan structure equation $d \sigma^{a}=\frac{1}{2} \epsilon^{a b c} \sigma^{b} \wedge \sigma^{c}$ given earlier. On the other hand, from our choice of SO(4)-symmetric ansätz for the YM field strength, one has $F^{a}=\frac{1}{2} F_{\mu \nu}^{a} d x^{\mu} \wedge d x^{\nu}=\frac{1}{2} F_{b c}^{a}\left(e^{b} \wedge e^{c}\right)=\frac{1}{2} \sigma^{2} a^{2}(\tau) f(\tau) \epsilon^{a b c}\left(e^{b} \wedge e^{c}\right)$. Then by equating these two alternative expressions for the field strength 2-form $F^{a}$, one gets a set of two equations

$$
\begin{gathered}
\partial_{\tau}\left[a(\tau) A_{b}^{a}(\tau)\right]=0, \\
A_{b}^{a}(\tau) \epsilon^{b d e}+\sigma a(\tau) A_{d}^{b}(\tau) A_{e}^{c}(\tau) \epsilon^{a b c}=\sigma a(\tau) f(\tau) \epsilon^{a d e} .
\end{gathered}
$$

Immediately, one can realize that these two equation can be simultaneously satisfied provided

$$
\begin{aligned}
A_{b}^{a}(\tau) & =\frac{k}{\sigma a(\tau)} \delta_{b}^{a}, \\
f(\tau) & =\frac{k(k+1)}{\sigma^{2} a^{2}(\tau)} .
\end{aligned}
$$

Our next job would then be to demonstrate that this choice of the $\mathrm{SO}(4)$-symmetric ansätz for YM gauge potential and its field strength tensor are legitimate since they indeed satisfy the Bianchi identity. As a matter of fact, it is straightforward to check that the Bianchi identity $D F^{a}=d F^{a}-\epsilon^{a b c} F^{b} \wedge A^{c}=0$ is satisfied with our choice of ansätz, $A^{a}=k \sigma^{a}$ and $F^{a}=\frac{1}{2} k(k+1) \epsilon^{a b c}\left(\sigma^{b} \wedge \sigma^{c}\right)$. Therefore, we can conclude that a consistent choice of the $\mathrm{SO}(4)$-symmetric ansätz for non-vanishing components of YM gauge potential and its field strength are

$$
\begin{aligned}
& A_{b}^{a}(\tau)=e_{b}^{\mu} A_{\mu}^{a}=\frac{k}{\sigma a(\tau)} \delta_{b}^{a}, \\
& F_{b c}^{a}(\tau)=e_{b}^{\mu} e_{c}^{\nu} F_{\mu \nu}^{a}=\frac{k(k+1)}{\sigma^{2} a^{2}(\tau)} \epsilon^{a b c} .
\end{aligned}
$$

Notice, again, that in contrast to the formulation employed in the original work by Hosoya and Ogura [5] where they obtained these types of expressions for YM gauge potential and 
its field strength tensor only after explicitly solving the classical YM field equation, here we obtained them simply from definitions and the Bianchi identity. Therefore, we particularly would like to stress that these $\mathrm{SO}(4)$-symmetric ansätz for $A^{a}$ and $F^{a}$ remain valid even off-shell as well as on-shell. In other words, these expressions for the YM gauge potential and its field strength can be used for both classical and quantum treatment that we shall discuss later on. As announced in the introduction, the aim of this work is to study the physics of classical and quantum wormholes in EYM theory. And we are supposed to study the classical wormhole solutions and their effects on low-energy physics in the present section and the quantum wormholes later on. To repeat, wormholes are Euclidean metrics which are solutions to the Euclidean classical field equations representing spacetimes consisting of two asymptotic Euclidean regions joined by a narrow tube or throat. Thus to study classical wormholes, we need to first look for exact solutions to the Euclidean classical field equations which exhibit above-mentioned geometrical structure. Note that we already have chosen SO(4)-symmetric ansätz for the YM gauge potential and its field strength which are consistent with the definitions and the Bianchi identity. Thus before we attempt to solve the Einstein equation, we need to check if these ansätz for YM fields can be solution to the classical YM field equation. As we shall see shortly, demanding that they be a solution amounts to fixing the parameter " $k$ " appearing in the ansätz. Now, since we choose to work in non-coordinate basis, we also consider the classical YM field equation in non-coordinate basis as well. And in non-coordinate basis, the YM field equation is obtained by simply replacing $F^{a}$ with its Hodge dual $\tilde{F}^{a}$ in the Bianchi identity equation given earlier, namely

$$
D \tilde{F}^{a}=d \tilde{F}^{a}-\epsilon^{a b c} \tilde{F}^{b} \wedge A^{c}=0 .
$$

Since the Hodge dual of the YM field strength is obtained to be

$$
\tilde{F}^{a}=k(k+1) \frac{N(\tau)}{a(\tau)}\left(d \tau \wedge \sigma^{a}\right)
$$

it is again straightforward to confirm that our choice of the $\mathrm{SO}(4)$ - symmetric YM gauge field given earlier does indeed satisfy the classical YM field equation provided $k=-1 / 2$, namely if 


$$
\begin{aligned}
& A^{a}=k \sigma^{a}=-\frac{1}{2} \sigma^{a}, \\
& F^{a}=\frac{1}{2} k(k+1) \epsilon^{a b c}\left(\sigma^{b} \wedge \sigma^{c}\right)=-\frac{1}{8} \epsilon^{a b c}\left(\sigma^{b} \wedge \sigma^{c}\right) .
\end{aligned}
$$

Next, we turn to the Einstein field equation. Since the SO(4)-symmetric ansätz for YM fields are given in terms of the scale factor $a(\tau)$, the coupled Einstein-YM field equations essentially reduce to the Einstein field equation of the scale factor $a(\tau)$ alone.

Now consider the $\tau \tau$-component of the Einstein field equation

$$
R_{\tau \tau}-\frac{1}{2} g_{\tau \tau} R+\frac{8 \pi}{M_{p}^{2}} \Lambda g_{\tau \tau}=\frac{8 \pi}{M_{p}^{2}} T_{\tau \tau}
$$

The YM field energy-momentum tensor in coordinate basis, $T_{\tau \tau}$, is related to its counterpart in non-coordinate basis by $T_{\tau \tau}=e_{\tau}^{A} e_{\tau}^{B} T_{A B}=e_{\tau}^{0} e_{\tau}^{0} T_{00}$ and $T_{00}$ can be easily computed by YM field strength in non-coordinate basis, $F_{0 b}^{a}=0, F_{b c}^{a}=\left(-\frac{1}{4}\right) \frac{1}{\sigma^{2} a^{2}(\tau)} \epsilon^{a b c}$ to be $T_{00}=$ $\left(-\frac{3}{32}\right) \frac{1}{\sigma^{4} g_{c}^{2} a^{4}(\tau)}$. Thus also using,

$$
\begin{aligned}
R_{\tau \tau} & =\left[-3\left(\frac{\ddot{a}}{a}\right)+3\left(\frac{\dot{N}}{N}\right)\left(\frac{\dot{a}}{a}\right)\right], \\
R & =\frac{6}{\sigma^{2}}\left[\left(\frac{\dot{N}}{N^{3}}\right)\left(\frac{\dot{a}}{a}\right)-\frac{1}{N^{2}}\left(\frac{\ddot{a}}{a}+\left(\frac{\dot{a}}{a}\right)^{2}\right)+\frac{1}{a^{2}}\right]
\end{aligned}
$$

where overdot denotes the derivative with respect to the Euclidean time $\tau$ and $\sigma^{2} \equiv 2 / 3 \pi M_{p}^{2}$, one gets the $\tau \tau$ - component of the Einstein equation as

$$
\left(\frac{d a}{d \tau}\right)^{2}=N^{2}-\lambda N^{2} a^{2}-N^{2} \frac{r_{0}^{2}}{a^{2}}
$$

where $r_{0}^{2} \equiv 3 \pi^{2} / 8 g_{c}^{2}$ and we introduced "dimensionless cosmological constant" as $\lambda \equiv$ $16 \Lambda / 9 M_{p}^{4}$. Now, we attempt to solve this Einstein field equation with different gauge choice for the lapse $N(\tau)$ and in the presence/absence of the cosmological constant.

\section{In the absence of the cosmological constant}

(i) With the gauge choice $N(\tau)=1$ :

The Einstein equation above reduces to

$$
\left(\frac{d a}{d \tau}\right)^{2}=1-\frac{r_{0}^{2}}{a^{2}}
$$


of which the solution is given by

$$
\tau=\int_{0}^{\tau} d \tau^{\prime}=\int_{a(0)=r_{0}}^{a} \frac{a d a}{\sqrt{a^{2}-r_{0}^{2}}}
$$

which yields upon the integration

$$
a(\tau)=\left[r_{0}^{2}+\tau^{2}\right]^{1 / 2}
$$

where we chose that the wormhole throat, i.e., the minimum value of $a(\tau)$ occurs for $\tau=0$, namely $\left.\frac{d a}{d \tau}\right|_{\tau=0}=0$ and $a(\tau=0)=r_{0}$. This solution represents a Tolman-type wormhole found first by Hosoya and Ogura [5].

(ii) With the gauge choice $N(\tau)=a(\tau)$ :

The Einstein equation, this time, reduces to

$$
\left(\frac{d a}{d \tau}\right)^{2}=a^{2}-r_{0}^{2}
$$

of which the solution is given by

$$
\tau=\int_{0}^{\tau} d \tau^{\prime}=\int_{a(0)=r_{0}}^{a} \frac{d a}{\sqrt{a^{2}-r_{0}^{2}}}
$$

which yields upon performing the integration

$$
a(\tau)=r_{0} \cosh (2 \tau)
$$

\section{In the presence of the cosmological constant}

(i) With the gauge choice $N(\tau)=1$ :

The Einstein equation reads

$$
\left(\frac{d a}{d \tau}\right)^{2}=1-\lambda a^{2}-\frac{r_{0}^{2}}{a^{2}}
$$

As usual, we choose the minimum value of $a(\tau)$, i.e., the wormhole neck to occur for $\tau=0$. Then from

$$
\left.\left(\frac{d a}{d \tau}\right)^{2}\right|_{\tau=0}=\frac{1}{a^{2}}\left[-\lambda a^{4}+a^{2}-r_{0}^{2}\right]=0
$$


which has zeros at $r_{ \pm}^{2}=\frac{1}{2 \lambda}\left[1 \pm \sqrt{1-4 \lambda r_{0}^{2}}\right]$, the radius of the wormhole neck, which exists when $\lambda<1 / 4 r_{0}^{2}$, is found to be

$$
a(0)=r_{-}=\left[\frac{1}{2 \lambda}\left(1-\sqrt{1-4 \lambda r_{0}^{2}}\right)\right]^{1 / 2}
$$

Thus, upon carrying out the integration

$$
\tau=\int_{0}^{\tau} d \tau^{\prime}=\int_{r_{-}}^{a} \frac{a d a}{\sqrt{-\lambda a^{4}+a^{2}-r_{0}^{2}}}
$$

one gets

$$
a(\tau)=\left[\frac{1}{2 \lambda}\left\{1-\sqrt{1-4 \lambda r_{0}^{2}} \cos (2 \sqrt{\lambda} \tau)\right\}\right]^{1 / 2} .
$$

This solution for the scale factor $a(\tau)$ takes the minimum value at $\tau=0, a_{\min }=\left[\frac{1}{2 \lambda}(1-\right.$ $\left.\left.\sqrt{1-4 \lambda r_{0}^{2}}\right)\right]^{1 / 2}$ and the maximum value at $\tau=\pi / 2 \sqrt{\lambda}, a_{\max }=\left[\frac{1}{2 \lambda}\left(1+\sqrt{1-4 \lambda r_{0}^{2}}\right)\right]^{1 / 2}$. Thus this solution represents a typical wormhole configuration.

(ii) With the gauge choice $N(\tau)=a(\tau)$ :

In this gauge, the Einstein equation reads

$$
\left(\frac{d a}{d \tau}\right)^{2}=a^{2}-\lambda a^{4}-r_{0}^{2}
$$

Once again, we choose the minimum value of $a(\tau)$, i.e., the wormhole neck to occur for $\tau=0$, i.e., $\left.\left(\frac{d a}{d \tau}\right)\right|_{\tau=0}=0$, then $a(0)=r_{-}=\left[\frac{1}{2 \lambda}\left(1-\sqrt{1-4 \lambda r_{0}^{2}}\right)\right]^{1 / 2}$ which is possible provided $\lambda<1 / 4 r_{0}^{2}$. Then using $\left(-\lambda a^{4}+a^{2}-r_{0}^{2}\right)=-\lambda\left(a^{2}-r_{+}^{2}\right)\left(a^{2}-r_{-}^{2}\right)$,

$$
\begin{aligned}
\tau=\int_{0}^{\tau} d \tau^{\prime} & =\int_{r_{-}}^{a} \frac{d a}{\sqrt{-\lambda a^{4}+a^{2}-r_{0}^{2}}} \\
& =\int_{r_{-}}^{a} \frac{d a}{\sqrt{-\lambda\left(a^{2}-r_{+}^{2}\right)\left(a^{2}-r_{-}^{2}\right)}}
\end{aligned}
$$

This integral can be expressed in terms of the elliptic integral and it is

$$
\sqrt{-\lambda} r_{+} \tau=F\left(\frac{a}{r_{-}}, \frac{r_{-}}{r_{+}}\right)-F\left(1, \frac{r_{-}}{r_{+}}\right)
$$

where $F(x, k)$ denotes the elliptic integral of the 1 st kind $(0<k<1)$ and $F(x=1, k) \equiv$ $K(k)$ is the "complete elliptic integral" of the 1st kind. 
Having constructed wormhole instanton solutions with different gauge choices for the lapse $N(\tau)$, we now turn to their contribution to the topology- changing tunnelling amplitude. Namely, we evaluate the wormhole instanton action $I_{E Y M}$ (instanton) (here $I_{E Y M}$ denotes the Euclidean action of the EYM theory), i.e., the minimum Euclidean action of the wormhole instanton configuration which makes dominant contribution to the topology-changing tunnelling amplitude. And this amounts to substituting the Einstein field equation (its trace) satisfied by the wormhole instanton solution

$$
R=\frac{32 \pi}{M_{p}^{2}} \Lambda
$$

into the Euclidean EYM theory action given earlier to obtain

$$
I_{E Y M}(\text { instanton })=\int d^{4} x \sqrt{g}\left[-\Lambda+\frac{1}{4 g_{c}^{2}}\left(F_{\mu \nu}^{a}\right)^{2}\right] .
$$

Then using, $\int d^{4} x \sqrt{g}=\left(2 \pi^{2} \sigma^{4}\right) \int_{0}^{\infty} d \tau N a^{3}, \sigma^{2}=2 / 3 \pi M_{p}^{2}, \lambda=16 \Lambda / 9 M_{p}^{4}$ and $\left(F_{\mu \nu}^{a}\right)^{2}=$ $\left(F_{b c}^{a}\right)^{2}=3 / 8 \sigma^{4} a^{4}$, this becomes

$$
\begin{aligned}
I_{E Y M}(\text { instanton }) & =\int_{0}^{\infty} d \tau N a^{3}\left[-\frac{8 \Lambda}{9 M_{p}^{4}}+\frac{3 \pi^{2}}{16 g_{c}^{2}} \frac{1}{a^{4}}\right] \\
& =\frac{1}{2} \int_{0}^{\infty} d \tau\left(\frac{N}{a}\right)\left[-\lambda a^{4}+r_{0}^{2}\right]=\frac{1}{2} \int_{R_{-}}^{a(\infty)} d a \frac{\left(-\lambda a^{4}+r_{0}^{2}\right)}{\sqrt{-\lambda a^{4}+a^{2}-r_{0}^{2}}}
\end{aligned}
$$

where we used the $\tau \tau$-component of the Einstein equation $(d a / d \tau)^{2}=N^{2}\left(1-\lambda a^{2}-r_{0}^{2} / a^{2}\right)$ which yields $d \tau(N / a)=d a / \sqrt{-\lambda a^{4}+a^{2}-r_{0}^{2}}$. Note, first, that above expression for $I_{E Y M}($ instanton $)$ is completely independent of the lapse function $N(\tau)$. Since the relevant gauge freedom for the case at hand is the arbitrariness in choosing the lapse $N(\tau)$, this means that the wormhole instanton action $I_{E Y M}$ (instanton) has gauge-invariance. As a matter of fact, since the quantity $\exp \left[-I_{E Y M}(\right.$ instanton $\left.)\right]$ represents the semi-classical approximation to the topology-changing tunnelling amplitude, it is a physical observable which should have manifest gauge- invariance. And we have just confirmed this point. Next note that unfortunately, it is not possible to carry out explicitly the integral in the expression for $I_{E Y M}$ (instanton) above to obtain the precise value of the wormhole instanton action. As was pointed out by Hosoya and Ogura [5], however, for a sufficiently small 
cosmological constant, this instanton action may be expanded in powers of the cosmological constant $\lambda=16 \Lambda / 9 M_{p}^{4}$ to yield

$$
I_{E Y M}(\text { instanton })=-\frac{1}{3 \lambda}-\frac{3 \pi}{4 \alpha_{g}} \ln \left(r_{0} \sqrt{\lambda}\right)+O(\sqrt{\lambda})
$$

where $r_{0}^{2}=3 \pi^{2} / 8 g_{c}^{2}$ as introduced earlier and $\alpha_{g} \equiv g_{c}^{2} / 4 \pi$. The first term results from the contribution from the half of the de Sitter instanton while the second term represents the contribution from the wormhole (neck) geometry especially with the logarithmic factor indicating the infrared cut-off generated by the finite wormhole neck size. We now end with few remarks. Firstly, note that the expression for $I_{E Y M}$ (instanton) given above in eq.(29) is valid only for sufficiently small values of the cosmological constant. Next, consider the contribution from the wormhole configuration to the topology- changing tunnelling amplitude

$$
\exp \left[\frac{3 \pi}{4 \alpha_{g}} \ln \left(r_{0} \sqrt{\lambda}\right)\right] .
$$

As Hosoya and Ogura [5] remarked, at first sight, this factor appears to serve as a suppression rather than an enhancement for the tunnelling amplitude since $\lambda$ is assumed to be small anyway. Besides, wormholes with larger size, namely, larger than the scale set by the dimensionless cosmological constant, i.e., $r_{0}>1 / \sqrt{\lambda}$ appears to enhance the tunnelling amplitude than the ones with smaller size. This last point is particularly disappointing since it is just against our expectation. As a matter of fact, it is noteworthy that just the opposite happens in the physics of axionic wormholes studied by Giddings and Strominger [4] where the tunnelling amplitude is highly suppressed for wormholes with size greater than the Planck length. All these arguments might be rather hasty since it is the whole quantity

$$
\exp \left[-I_{E Y M}(\text { instanton })\right]=e^{\frac{1}{3 \lambda}}\left(r_{0} \sqrt{\lambda}\right)^{\frac{3 \pi}{4 \alpha_{g}}}
$$

that one really should consider as the right tunnelling amplitude. And for sufficiently small $\lambda$, the first singular factor dominates over the second factor (coming from the wormhole contribution) still producing a sizable tunnelling amplitude. Neverthless, since it is the role 
played by the small cosmological constant that essentially elevates the tunnelling amplitude to a sizable magnitude, it seems fair to say that the wormhole instanton solutions in this EYM theory do not seem to be local minima of the Euclidean action which have manifest contribution to the semiclassical tunnelling amplitude.

\section{Quantum Wormholes in Einstein-Yang-Mills Theory}

Thus far, we have considered classical treatment of wormholes in EYM theory. Namely we constructed $\mathrm{SO}(4)$-symmetric metric solutions to the classical EYM field equations that exhibit the geometry of wormhole spacetimes and studied their nature. We now turn to the quantum treatment of wormholes in the same EYM theory. The formulation we shall employ can be summarized as follows : we construct and study a minisuperspace model (again based on $\mathrm{SO}(4)$-symmetry) of canonical quantum cosmology in which the main objective is to solve the Wheeler-DeWitt (WD) equation to find the universe wave function. And to see if there is an excitation that can be interpreted as a "quantum wormhole", we look for a particular solution to the WD equation with "wormhole boundary condition". Here the wormhole boundary condition refers to an appropriate boundary condition of a universe wave function that allows one to naturally identify the universe wave function as representing an excitation corresponding to a wormhole state. And as a proposal for such wormhole boundary condition, we shall employ the one advocated by Hawking and Page. According to their proposal, wormhole wave functions are supposed to behave in such a way that they are damped, say, exponentially, for large 3 -geometries $(\sqrt{h} \rightarrow \infty)$ and are regular in some suitable way when the 3 -geometry collapses to zero $(\sqrt{h} \rightarrow 0)$. We now begin by constructing a minisuperspace quantum cosmology model based on EYM theory described by the action given earlier. As already stated, to do so, we choose to take the avenue of canonical quantum cosmology based on Arnowitt-Deser-Misner (ADM)'s (3+1)- space-plustime split formalism [11-13]. As usual, then, in order to render the system tractable, we reduce the infinite-dimensional "superspace" down to a finite-dimensional minisuperspace by assuming that the 4 -dim. spacetime has the geometry of spatially-closed $(k=+1)$ FRWmetric which is the one we adopted in the classical treatment of the system earlier. The 
geometry of its spatial section is, then, that of $S^{3}$ and hence it posseses $\mathrm{SO}(4)$-symmetry. Now, since the spacetime geometry is taken to possess the $\mathrm{SO}(4)$-symmtry, the matter field defined on it, i.e., the YM gauge field should have the same SO(4)-symmetry. Thus the $\mathrm{SO}(4)$-symmetric ansätz for the metric is again the spatially-closed FRW-metric

$$
\begin{aligned}
d s^{2} & =\sigma^{2}\left[N^{2}(\tau) d \tau^{2}+a^{2}(\tau) \sigma^{a} \otimes \sigma^{a}\right] \\
& =\eta_{A B} e^{A} \otimes e^{B}
\end{aligned}
$$

and the $\mathrm{SO}(4)$-symmetric ansätz for the YM gauge potential and its field strength tensor are, in non-coordinate basis

$$
\begin{array}{ll}
A_{0}^{a}(\tau)=0, & A_{b}^{a}(\tau)=\frac{k}{\sigma a(\tau)} \delta_{b}^{a} \\
F_{0 b}^{a}(\tau)=0, & F_{b c}^{a}(\tau)=\frac{k(k+1)}{\sigma^{2} a^{2}(\tau)} \epsilon^{a b c}
\end{array}
$$

where $A, B=0,1,2,3$ and $a, b, c=1,2,3$ and again $\sigma^{2}=2 / 3 \pi M_{p}^{2}$. Note first that in the metric, if $N$ is imaginary, it is the Lorentzian metric with Lorentzian time $\tau=t$ and if $N$ real, it is the Euclidean metric with Euclidean time $\tau$. As is well-known and as we shall see later, the WD equation and its solution are independent of $N$ and $\tau$ and hence remain the same in both the Lorentzian and Euclidean signatures. Next, recall that the $\mathrm{SO}(4)$-symmetric ansätz for $A_{B}^{a}(\tau)$ and $F_{B C}^{a}(\tau)$ given above remain valid off-shell as well as on-shell as we stressed in the classical treatment of the system discussed earlier. And it is because we obtained them simply from definitions and the Bianchi identity which are bottomline conditions that should be met in quantum formulations as well. Thus in the present quantum treatment, the parameter $k$ appearing in the ansätz for YM gauge field are undetermined. (As we have seen, it gets determined to be $k=-1 / 2$ only when one imposes on-shell condition, namely only if one demands the classical YM field equation to be satisfied.) Now, for the sake of definiteness, we choose the Euclidean signature (i.e., $N(\tau)$ is real) and write the Euclidean action of the EYM theory given earlier in terms of these $\mathrm{SO}(4)$-symmetric ansätz for the metric and YM gauge field. Namely, using $\int d^{4} x \sqrt{g}=\int d \tau N\left(\int_{S^{3}} d^{3} x \sqrt{h}\right)=\left(2 \pi^{2} \sigma^{4}\right) \int d \tau N a^{3}$ and 


$$
\begin{aligned}
R & =\frac{6}{\sigma^{2}}\left[\left(\frac{\dot{N}}{N^{3}}\right)\left(\frac{\dot{a}}{a}\right)-\frac{1}{N^{2}}\left(\frac{\ddot{a}}{a}+\left(\frac{\dot{a}}{a}\right)^{2}\right)+\frac{1}{a^{2}}\right], \\
\left(F_{\mu \nu}^{a}\right)^{2} & =\left(F_{b c}^{a}\right)^{2}=6 \frac{k^{2}(k+1)^{2}}{\sigma^{4} a^{4}(\tau)}
\end{aligned}
$$

with the overdot denoting the derivative with respect to $\tau$, the Euclidean action of EYM theory takes the form

$$
\begin{aligned}
I_{G} & =\int d^{4} x \sqrt{g}\left[\Lambda-\frac{M_{p}^{2}}{16 \pi} R\right]=\frac{1}{2} \int d \tau N a^{3}\left[\lambda-\left\{\frac{1}{a^{2}}+\left(\frac{\dot{a}}{N a}\right)^{2}\right\}\right], \\
I_{Y M} & =\int d^{4} x \sqrt{g}\left[\frac{1}{4 g_{c}^{2}}\left(F_{\mu \nu}^{a}\right)^{2}\right]=\left(2 \pi^{2} \sigma^{4}\right) \int d \tau N a^{3}\left[\frac{1}{4 g_{c}^{2}}\left\{6 \frac{k^{2}(k+1)^{2}}{\sigma^{4} a^{4}}\right\}\right] \\
& =\frac{1}{2} \int d \tau N a^{3}\left[\frac{r_{0}^{2}}{a^{4}}\right]
\end{aligned}
$$

and hence

$$
\begin{aligned}
I_{E Y M} & =I_{G}+I_{Y M} \\
& =\frac{1}{2} \int d \tau N a^{3}\left[\lambda-\left\{\frac{1}{a^{2}}+\left(\frac{\dot{a}}{N a}\right)^{2}\right\}+\frac{r_{0}^{2}}{a^{4}}\right] .
\end{aligned}
$$

For the sake of completeness we also provide the Lorentzian action of the EYM theory which can be obtained via $i S_{E Y M}=-I_{E Y M}$ and $\tau=i t$

$$
S_{E Y M}=\frac{1}{2} \int d t N a^{3}\left[-\lambda+\left\{\frac{1}{a^{2}}-\left(\frac{\dot{a}}{N a}\right)^{2}\right\}-\frac{r_{0}^{2}}{a^{4}}\right] \equiv \int d t L_{A D M}
$$

where now the overdot denotes the derivative with respect to the Lorentzian time $t$ and again $\lambda$ denotes the dimensionless cosmological constant $\lambda=16 \Lambda / 9 M_{p}^{4}$ and $r_{0}^{2} \equiv k^{2}(k+1)^{2} 6 \pi^{2} / g_{c}^{2}$ which becomes its classical counterpart $r_{0}^{2}=3 \pi^{2} / 8 g_{c}^{2}$ for $k=-1 / 2$. Now the subsequent procedure toward the derivation of the WD equation via the Legendre transformation to the classical Hamiltonian and the Dirac quantization can be best demonstrated in familiar Lorentzian signature. Thus from this point on we work with the Lorentzian action for the construction of the WD equation. Before we proceed, however, here we make one important remark on the structure of the EYM theory action written in SO(4)-symmetric ansätz given above. The fact that we choose to consider only the magnetically-charged case $\left(F_{0 b}^{a}=0\right)$ and the fact that the $\mathrm{SO}(4)$-symmetric ansätz for YM field strength is written as $F_{b c}^{a}=\frac{k(k+1)}{\sigma^{2} a^{2}} \epsilon^{a b c}$ 
effectively reduce the EYM theory action to the action of a single minisuperspace variable, i.e., the scale factor $a(\tau)$ alone. Thus the resulting WD equation will also become a one-dim. Schrödinger-type equation for the universe wave function $\Psi[a]$. As we shall see shortly, this reduction to one-dim. problem of minisuperspace model essentially makes it possible to find the universe wave function which represents a quantum wormhole state. Formally speaking, this reduction is achieved by the full gauge-fixing which consists of the gauge choice $N^{i}=0$ associated with the 3 -dim. diffeomorphism in the gravity sector and the gauge choice $A_{0}^{a}=0$ in the YM sector.

Now getting back to the derivation procedure for the WD equation, we identify the canonical momentum conjugate to the minisuperspace variable $a$ with

$$
p_{a}=\frac{\partial L_{A D M}}{\partial \dot{a}}=\frac{a}{N}(-\dot{a})
$$

and then obtain the classical Hamiltonian via the Legendre transformation

$$
\begin{aligned}
S_{E Y M} & =\int d t L_{A D M} \\
& =\int d t\left(p_{a} \dot{a}-H_{A D M}\right)=\int\left[p_{a} d a-\left(N H_{0}+N_{i} H^{i}\right) d t\right]
\end{aligned}
$$

where $N_{i}=0$ and $H_{A D M}=N H_{0}+N_{i} H^{i}$ denotes the ADM Hamiltonian consisting of the sum of secondary constraints $H_{0}=0$ and $H^{i}=0$ as we shall discuss shortly. Namely the classical Hamiltonian is obtained as

$$
\begin{aligned}
\frac{\delta S_{E Y M}}{\delta N} & =\frac{1}{2} a^{3}\left[-\lambda+\frac{1}{a^{2}}+\frac{\dot{a}^{2}}{N^{2} a^{2}}-\frac{r_{0}^{2}}{a^{4}}\right] \\
& =\frac{1}{2 a}\left[p_{a}^{2}-\lambda a^{4}+a^{2}-r_{0}^{2}\right] .
\end{aligned}
$$

Namely,

$$
\begin{aligned}
H_{0}=-\frac{\delta S_{E Y M}}{\delta N} & =\frac{1}{2 a}\left[-p_{a}^{2}+\left(\lambda a^{4}-a^{2}+r_{0}^{2}\right)\right] \\
& \equiv \frac{1}{2 a}\left[-p_{a}^{2}+U(a)\right] \\
\text { where } U(a) & =\lambda a^{4}-a^{2}+r_{0}^{2} .
\end{aligned}
$$

General relativity is one of the most well-known constrained system. The invariance of the system under the 4-dim. diffeomorphisms (consisting of the time-reparametrization 
and the 3-dim. general coordinate transformations of the spacelike hypersurface) leads to the emergence of 4-constraint equations. Of them, we need not explicitly impose the 3-momentum constraint equations since we already have taken the $N^{i}=0$ gauge which amounts to assuming the $S O(4)$-symmetric spatially-closed FRW-metric. Thus, we only need to impose the Hamitonian constraint $H_{0}=0$. The classical Hamiltonian constraint now reads

$$
H_{0}=\frac{1}{2 a}\left[-p_{a}^{2}+\left(\lambda a^{4}-a^{2}+r_{0}^{2}\right)\right]=0
$$

Next, in order to quantize this EYM system, we need to turn to the "Dirac quantization procedure" for the constrained system. According to the Dirac quantization procedure, the invariance in the action of the theory under the 4-dim. diffeomorphisms is secured by demanding that the physical (universe) wave function $\Psi$ be annihilated by "operator versions" of the 4-constraints. Therefore, the classical Hamitonian constraint above turns into its quantum version, namely the WD equation given by

$$
\hat{H}_{0}\left(p_{a}=-i \frac{\partial}{\partial a}\right) \Psi[a]=0
$$

In order to obtain the correct form of this WD equation, we first examine the structure of the classical Hamiltonian

$$
\begin{aligned}
H_{0} & =\frac{1}{2 a}\left[-p_{a}^{2}+\left(\lambda a^{4}-a^{2}+r_{0}^{2}\right)\right] \\
& =T+V \equiv \frac{1}{2} G^{\alpha \beta} \Pi_{\alpha} \Pi_{\beta}+V
\end{aligned}
$$

where $V=\frac{1}{2 a} U(a)=\frac{1}{2 a}\left(\lambda a^{4}-a^{2}+r_{0}^{2}\right)$. Here, one can readily read off the "minisuperspace metric" $G_{\alpha \beta}$ as

$$
G_{\alpha \beta}=-a \delta_{\alpha \beta} \quad, \quad G^{\alpha \beta}=-\frac{1}{a} \delta^{\alpha \beta}
$$

with $\gamma^{\alpha}=a$ and $\Pi_{\alpha}=p_{a}$ being the minisuperspace variable and its conjugate momentum respectively. Then, by the usual substitution,

$$
G^{\alpha \beta} \Pi_{\alpha} \Pi_{\beta} \quad \rightarrow \quad-\nabla^{2}
$$


with $\nabla^{2}=\frac{1}{\sqrt{G}} \frac{\partial}{\partial \gamma^{\alpha}}\left(\sqrt{G} G^{\alpha \beta} \frac{\partial}{\partial \gamma^{\beta}}\right)=-\frac{1}{a} \frac{\partial}{\partial a}\left(\frac{\partial}{\partial a}\right)$, finally we arrive at the WD equation

$$
\hat{H}_{0} \Psi=\frac{1}{2}\left[\frac{1}{a} \frac{\partial}{\partial a}\left(\frac{\partial}{\partial a}\right)+\frac{1}{a} U(a)\right] \Psi[a]=0 .
$$

Note, here, that the minisuperspace metric (or "DeWitt metric") $G_{\alpha \beta}(\gamma)$ is generally a function of minisuperspace variables $\gamma^{\alpha}$. Therefore, in passing from classical to quantum version, there arises the "ambiguity in operator ordering" problem. And as have been suggested by Hartle and Hawking [12], one may partly encompass this "operator-ordering" problem by rewritting

$$
\frac{\partial}{\partial a}\left(\frac{\partial}{\partial a}\right) \quad \rightarrow \quad \frac{1}{a^{p}} \frac{\partial}{\partial a}\left(a^{p} \frac{\partial}{\partial a}\right)
$$

Finally, the WD equation takes the general form

$$
\frac{1}{2}\left[\frac{1}{a^{p}} \frac{\partial}{\partial a}\left(a^{p} \frac{\partial}{\partial a}\right)+U(a)\right] \Psi[a]=0
$$

where " $p$ " denotes a suffix representing the ambiguity in operator-ordering. Before we go on and attempt to solve this WD equation explicitly, we would like to have some insight into the behavior of the solution of this WD equation. To do so, we begin by assigning the "normal" sign to the "kinetic" energy term to get

$$
\frac{1}{2}\left[\frac{-1}{a^{p}} \frac{\partial}{\partial a}\left(a^{p} \frac{\partial}{\partial a}\right)+\tilde{U}(a)\right] \Psi[a]=0 .
$$

Then the "potential" energy can be identified with

$$
\tilde{U}(a)=-U(a)=\left(a^{2}-\lambda a^{4}-r_{0}^{2}\right) .
$$

The Fig. 1(2) given displays the plot of potential energy as it appears in the WD equation in the presence (absence) of the cosmological constant $\lambda=16 \Lambda / 9 M_{p}^{4}$. Note here that $\tilde{U}(a)=\left(a^{2}-\lambda a^{4}-r_{0}^{2}\right)=0$ possesses two positive roots $R_{ \pm}$provided $\lambda<1 / 4 r_{0}^{2}$ or $\Lambda<$ $\left(\frac{g_{c}}{8 \pi}\right)^{2} \frac{3}{2 k^{2}(k+1)^{2}} M_{p}^{4}$. Both figures show that due to the contribution to the potential energy, $\left(-r_{0}^{2}\right)$, coming from the YM gauge field sector of the theory, the potential develops a "well" in the small- $a$ region for both cases with or without the cosmological constant term. Since 
the WD equation implies that the total energy of the gravity-matter system is zero, $E=0$, the emergence of the potential well in the small- $a$ region reveals the fact that the universe wave function $\Psi[a]$ should be an oscillating function of $a$ there. And this small- $a$ behavior of the universe wave function appears to signal the existence of "quantum wormhole" as well as other types of spacetime fluctuations in the small- $a$ region of the minisuperspace and hence seems consistent with the existence of classical wormhole solution in this EYM theory as we have seen in the earlier section. Obviously, the most straightforward way of confirming the possible existence of "quantum wormholes" is to solve the WD equation given above for the universe wave function which particularly represents an excitation corresponding to a wormhole state. Unfortunately, exact, analytic solutions to the WD equation in the presence of the cosmological constant are not available. (As a matter of fact, exact solutions to the WD equation even for de Sitter spacetime pure gravity are not available either $[12,13]$.) However, since our major concern is to find the wormhole wave function that is expected to exist within the potential well in the small- $a$ region, first we focus on the case in which the cosmological constant is absent and thus the potential energy takes the behavior depicted in Fig.2. Namely we consider the WD equation in the absence of the cosmological constant

$$
\left[\frac{\partial^{2}}{\partial a^{2}}+\frac{p}{a} \frac{\partial}{\partial a}-a^{2}+r_{0}^{2}\right] \Psi[a]=0
$$

Even in this simpler case, exact, analytic solutions to this WD equation which is valid for the whole range of $a,[0, \infty)$ are not available. However, this is not so disappointing since in order to see if this WD equation really admits a solution that can be interpreted as a wormhole wave function satisfying, say, the Hawking and Page-type wormhole boundary conditions, it suffices for us to know just the asymptotic behaviors (such as those at small- $a$ or at large- $a$ ) of the solution. Thus in the following we consider the behavior of the solution in the small- $a$ region and in the large- $a$ region (relative to the scale $r_{0}$ set by the dimensionless coupling parameter of the theory, i.e., $\left.r_{0}^{2}=k^{2}(k+1)^{2} 6 \pi^{2} / g_{c}^{2}\right)$.

(i) For $a<<r_{0}$ :

In this small- $a$ region, the WD equation in the absence of the cosmological constant above 
can be approximated by

$$
\left[\frac{\partial^{2}}{\partial a^{2}}+\frac{p}{a} \frac{\partial}{\partial a}+r_{0}^{2}\right] \Psi[a] \approx 0
$$

which admits an analytic solution given by

$$
\Psi\left[a<<r_{0}\right]=a^{\left(\frac{1-p}{2}\right)} J_{\left(\frac{1-p}{2}\right)}\left(r_{0} a\right)
$$

Note that this solution in the small- $a$ region is regular at $a=0$ provided $p<1$ and it represents an oscillating solution.

(ii) For $a>>r_{0}$ :

In this large- $a$ region, the WD equation above reduces to

$$
\left[\frac{\partial^{2}}{\partial a^{2}}+\frac{p}{a} \frac{\partial}{\partial a}-a^{2}\right] \Psi[a] \approx 0
$$

which admits an analytic solution given by

$$
\Psi\left[a>>r_{0}\right]=a^{\left(\frac{1-p}{2}\right)} J_{\left(\frac{1-p}{4}\right)}\left(\frac{i}{2} a^{2}\right)
$$

Note that as $a \rightarrow \infty, \Psi\left[a>>r_{0}\right] \rightarrow a^{-\left(\frac{1+p}{2}\right)} e^{ \pm \frac{1}{2} a^{2}}$, thus this solution does involve a damping solution in the large- $a$ region.

Namly the solution to the WD equation in the absence of the cosmological constant possesses regular, oscillating behavior in the classically-allowed region, $a<<r_{0}$, while it damps rapidly enough in the classically-forbidden region, $a>>r_{0}$ in accordance with our expectation. Then next, in order to see if these asymptotic behaviors of the solution are consistent with or equivalent to the "wormhole boundary condition" and hence allows us to identify the solution with the wave function of a quantum wormhole, we now refer to a wormhole boundary condition advocated by Hawking and Page [10]. Hawking and Page argued in a convincing manner that in order for a solution to a WD equation to represent quantum wormholes, it should obey certain boundary conditions. And the appropriate boundary conditions for wormhole wave functions seem to be that they are damped, say, expnentially for large 3 -geometries $(\sqrt{h} \rightarrow \infty)$ and are regular in some suitable way when the 3-geometry 
collapses to zero $(\sqrt{h} \rightarrow 0)$. Particularly, in the context of FRW minisuperspace model, large 3-geometries correspond to $a \rightarrow$ large limit and the 3-geometry collapsing to zero corresponds to $a \rightarrow 0$ limit. And the damping behavior of the universe wave function at large- $a$ indicates that there are no gravitational excitations asymptotically and hence it represents asymptotically-Euclidean spacetime while its regularity at $a=0$ indicates that it is nonsingular. Therefore, the solution to a WD equation obeying these boundary conditions must correspond to wormholes that connect two asymptotically-Euclidean regions. As we already have observed, since our solution to the WD equation in the absence of the cosmological constant is indeed regular at $a=0$ (provided $p<1$ ) and damps sufficiently rapidly at large- $a$, it does satisfy the "wornhole boundary condition". Namely the WD equation in the absence of the cosmological constant does admit wormhole wave function as its solution and most probably this will hold true for the WD equation in the presence of the cosmological constant. And this is because the essential point that allows the existence of the wormhole wave function in the small- $a$ region is the contribution to the potential energy, $\left(-r_{0}^{2}\right)$, coming from the YM gauge field sector of the theory as we mentioned earlier. Therefore, now we can conclude that there are quantum wormholes as well as classical wormhole solutions in EYM theory with or without the cosmological constant.

Next we go back to the case where the cosmological constant is present. We now would like to point out that the EYM theory with the cosmological constant may serve as an interesting simple model which provides an overall picture of universe's entire history from the deep quantum domain all the way to the typical, classical domain. To this end, first note that our knowledge on the nature of the universe wave function in the absence of the cosmological constant developed thus far may, in turn, enables us to construct the solution to the WD equation in the presence of the cosmological constant at least approximately yet quite systematically. Let us go back and consider the WD equation in EYM theory in the presence of the cosmological constant given earlier

$$
\frac{1}{2}\left[-\frac{1}{a^{p}} \frac{\partial}{\partial a}\left(a^{p} \frac{\partial}{\partial a}\right)+\left(a^{2}-\lambda a^{4}-r_{0}^{2}\right)\right] \Psi[a]=0 .
$$


With the potential energy, $\tilde{U}(a)=\left(a^{2}-r_{0}^{2}\right)$, as it appears in the WD equation in the absence of the cosmological constant, we now know that the solution to the WD equation represents a quantum wormhole states with oscillating behavior in the small- $a$ region. Therefore this observation plus the shape of the full potential energy, $\tilde{U}(a)=\left(a^{2}-\lambda a^{4}-r_{0}^{2}\right)$ in the presence of the cosmological constant as was depicted in Fig.1 suggest that the solution to the WD equation above would describe the state of the universe that undergoes "spacetime fluctuations for very small-a" $\rightarrow$ "spontaneous nucleation (quantum tunnelling) of the universe from nothing in a de Sitter geometry" $\rightarrow$ "subsequent, mainly classical evolution of the universe for large- $a "$. Thus if we are willing to accept $(\partial / \partial a)$ as the timelike killing field in the (mini)superspace, the EYM theory with the non-vanishing cosmological constant appears to serve as a simple yet interesting model which provides a comprehensive overall picture of entire universe's history from the deep quantum domain all the way to the essentially classical domain. Then coming back to a practical problem, now we wish to construct the approximate solutions to the WD equation. Roughly, the behavior of the solution for very small- $a$ will be determined by the wormhole wave function obtained in the present work while the behavior for intermediate-to-large- $a$ regions will be approximately governed by the de Sitter space universe wave function. Here, the de Sitter space universe wave functions have been constructed and extensively studied in the literature associated with the issue of initial or boundary conditions for the universe wave function. Therefore in order to employ the de Sitter space universe wave function, here we give a brief account of the issue of initial or boundary conditions for the universe wave function. The WD equation is a second order hyperbolic functional differential equation describing the evolution of the universe wave function in the superspace. Thus the WD equation, in general, has a large number of solutions and in order to have any predictive power, one needs initial or boundary conditions to pick out just one solution by, for instance, giving the value of the universe wave function at the boundary of the superspace. Thus far, a number of different proposals for the law of initial or boundary conditions have been put forward. And among 
them, "no-boundary proposal" of Hartle and Hawking (HH) [12] and "tunnelling boundary condition" due to Vilenkin [13] are the ones which are the most comprehensive and the most extensively studied. If stated briefly, the no boundary proposal by HH [12] is based on the philosophy that the quantum state of the universe is the closed cosmology's version of "ground state" or "state of minimum excitation" and the wave function of this ground state is given by an Euclidean sum-over-histories. Next, Vilenkin's tunnelling boundary condition [13] can be best stated in "outgoing modes" formulation which governs the behavior of the solutions to the WD equation at boundaries of the superspace. Namely, according to this proposal, at "singular" boundaries (such as the region of zero 3-metric and infinite 3-curvature $(\sqrt{h} \rightarrow 0))$ of superspace, the universe wave function should consist solely of outgoing modes carrying flux out of superspace. These two proposals for the law of initial or boundary conditions on the universe wave function determine the behaviors of the universe wave function like how the universe nucleated (from "nothing") and then following which line it has subsequently evolved to the present one. Indeed in the minisuperspace models and within the context of the semiclassical approximation, these two proposals have been successfully applied to and tested for simple systems such as de Sitter spacetime in pure gravity which is of our interest here or a scalar field theory coupled to gravity.

Thus employing the well-known de Sitter space universe wave functions with the choice of HH's no boundary proposal or Vilenkin's tunnelling boundary condition, we now construct the approximate solutions to the WD equation. And to do so we consider the WD equation in two regions of interest in the minisuperspace.

Firstly for very small- $a$ region, the WD equation reduces to eq.(51). As we have investigated earlier, the important asymptotic behaviors of the solution to this equation are given by

$$
\begin{aligned}
\Psi_{I}[a] & \rightarrow a^{\left(\frac{1-p}{2}\right)} J_{\left(\frac{1-p}{2}\right)}\left(r_{0} a\right) \quad(\text { for } \quad a \rightarrow 0) \\
& \rightarrow a^{\left(\frac{1-p}{2}\right)} J_{\left(\frac{1-p}{4}\right)}\left(\frac{i}{2} a^{2}\right) \rightarrow a^{-\left(\frac{1+p}{2}\right)} e^{ \pm \frac{1}{2} a^{2}} \quad \quad(\text { for } \quad a \rightarrow \text { large) }
\end{aligned}
$$

where $p<1$ for regularity of the solution. Secondly for intermediate-to-large- $a$ regions, the WD equation can be roughly approximated by 


$$
\left[\frac{\partial^{2}}{\partial a^{2}}+\frac{p}{a} \frac{\partial}{\partial a}-a^{2}+\lambda a^{4}\right] \Psi[a]=0 .
$$

As mentioned, the semiclassical approximation to the solutions of this de Sitter space WD equation has been thoroughly studied. First HH's no-boundary wave function [12] is given by

$$
\begin{aligned}
\Psi_{I I}^{H H}[a]= & a^{-\left(\frac{1+p}{2}\right)} \exp \left[\frac{1}{3 \lambda}\left\{1-\left(1-\lambda a^{2}\right)^{3 / 2}\right\}\right] \quad\left(\text { for } \quad \lambda a^{2}<1\right) \\
& \rightarrow a^{-\left(\frac{1+p}{2}\right)} e^{\frac{1}{2} a^{2}} \quad\left(\lambda a^{2}<<1\right), \quad\left(\text { for } \quad \lambda a^{2}>1\right) \\
= & a^{-\left(\frac{1+p}{2}\right)} \exp \left[\frac{1}{3 \lambda}\right] 2 \cos \left[\frac{\left(\lambda a^{2}-1\right)^{3 / 2}}{3 \lambda}-\frac{\pi}{4}\right] \quad \\
& \rightarrow a^{-\left(\frac{1+p}{2}\right)}\left[e^{i \frac{\sqrt{\lambda}}{3} a^{3}}+e^{-i \frac{\sqrt{\lambda}}{3} a^{3}}\right] \quad\left(\lambda a^{2}>>1\right) .
\end{aligned}
$$

This HH's no-boundary wave function consists of both "ingoing" (contracting) and "outgoing" (reexpanding) modes in the classical-allowed region $\left(\lambda a^{2}>1\right)$ which, then, decreases exponentially as it moves towards smaller values of $a$ in the classically-forbidden region $\left(\lambda a^{2}<1\right)$. Next, Vilenkin's tunnelling wave function [13] is given by

$$
\begin{aligned}
\Psi_{I I}^{T}[a]= & a^{-\left(\frac{1+p}{2}\right)}\left(1-\lambda a^{2}\right)^{-1 / 4} \exp \left[-\frac{1}{3 \lambda}\left\{1-\left(1-\lambda a^{2}\right)^{3 / 2}\right\}\right] \quad\left(\text { for } \quad \lambda a^{2}<1\right) \\
& \rightarrow a^{-\left(\frac{1+p}{2}\right)} e^{-\frac{1}{2} a^{2}} \quad\left(\lambda a^{2}<<1\right) \\
= & a^{-\left(\frac{1+p}{2}\right)} e^{i \frac{\pi}{4}}\left(\lambda a^{2}-1\right)^{-1 / 4} \exp \left[-\frac{1}{3 \lambda}\left\{1+i\left(\lambda a^{2}-1\right)^{3 / 2}\right\}\right] \quad\left(\text { for } \quad \lambda a^{2}>1\right)
\end{aligned}
$$

This Vilenkin's tunnelling wave function exponentially decreases as it moves from small toward larger values of $a$ (i.e., emerges out of the potential barrier via "quantum tunnelling") and then upon escaping the barrier, it consists solely of "outgoing" (expanding) mode in the classically-allowed region $\left(\lambda a^{2}>1\right)$. Now we are ready to write down approximate solutions to the WD equation in the presence of the cosmological constant by putting these pieces altogether. To do so let us denote the smaller and larger roots of the equation

$$
\tilde{U}(a)=a^{2}-\lambda a^{4}-r_{0}^{2}=0
$$

by $r_{-}$and $r_{+}$respectively which exist provided $\lambda<1 / 4 r_{0}^{2}$ or equivalently $\Lambda<\frac{3}{2}\left[\frac{g_{c}}{8 \pi k(k+1)}\right]^{2} M_{p}^{4}$. Then the two roots are given by $r_{ \pm}^{2}=\frac{1}{2 \lambda}\left[1 \pm \sqrt{1-4 \lambda r_{0}^{2}}\right]$.

(1) With the choice of HH's no-boundary wave function : 


$$
\begin{array}{rlrl}
\Psi[a] & =\Psi_{I}[a] \quad & \left(\text { region } I: 0<a<r_{+}\right), \\
& =\Psi_{I I}^{H H}[a] \quad\left(\text { region } I I: r_{-}<a<\infty\right) .
\end{array}
$$

(2) With the choice of Vilenkin's tunnelling wave function :

$$
\begin{aligned}
\Psi[a] & =\Psi_{I}[a] & & \left(\text { region } I: 0<a<r_{+}\right), \\
& =\Psi_{I I}^{T}[a] \quad & & \left(\text { region } I I: r_{-}<a<\infty\right) .
\end{aligned}
$$

The two kinds of universe wave functions corresponding to the two different choices of the boundary conditions are plotted in Fig 3 . and 4 respectively.

\section{Discussions}

Now we summarize the motivation and the results of the present work.

We revisited, in this work, the Einstein-Yang-Mills theory considered first by Hosoya and Ogura [5] which is one of the classic systems known to admit classical, Euclidean wormhole instanton solution. The classical wormhole instanton in this theory as a solution to the classical field equations and much of its effects on low energy physics have been studied extensively in the literature. Since this EYM system admits classical wormhole solutions, one may wonder if there is any systematic way of exploring the existence and the physics of "quantum" wormholes in the same theory. The present work attempted to deal with this kind of yet unquestioned issue. In order to explore the quantum wormholes in this system systematically, we worked in the context of canonical quantum cosmology and followed Hawking and Page [10] to define the quantum wormhole as a state or an excitation represented by a solution to the Wheeler-DeWitt equation satisfying a certain wormhole boundary condition. Particularly, in the minisuperspace quantum cosmology model possessing SO(4)symmetry, it is demonstrated that there indeed exists a solution to the Wheeler-DeWitt equation whose asymptotic behaviors satisfy the appropriate wormhole boundary condition for the case when the cosmological constant is absent. Thus we confirmed our expectation that the EYM system does admit quantum wormholes as well as classical wormholes. Further, we pointed out that the minisuperspace quantum cosmology model based on this EYM 
theory in the presence of the cosmological constant may serve as an simple yet interesting system displaying an overall picture of entire universe's history from the deep quantum domain all the way to the classical domain.

As we have stressed in the text, the essential point that allowed us to explore, in a concrete manner, the quantum wormhole in the context of the minisuperspace quantum cosmology model was the following observation. In their original work, Hosoya and Ogura [5] obtained the expressions for the YM gauge potential and its field strength tensor only after explicitly solving the classical YM field equation. In particular, the SO(4)-symmetric YM field strength tensor obtained in this way, $F_{0 b}^{a}=0, F_{b c}^{a}=\frac{k(k+1)}{\sigma^{2} a^{2}(\tau)} \epsilon^{a b c}$, in turn, reduces the Einstein equation to that of the scale factor $a(\tau)$ alone. However, we realized in this work that even without imposing the on-shell condition (i.e., the classical field equation), one can "derive" $F_{b c}^{a}=\frac{\text { const. }}{a^{2}} \epsilon^{a b c}$ just from the definitions and the Bianchi identity. Therefore this SO(4)-symmetric ansätz for the YM field strength $F_{b c}^{a}$ remains valid even off-shell as well as on-shell and hence can be used in the quantum treatment of the EYM system. Consequently, the Wheeler-DeWitt equation in the context of the canonical quantum cosmology becomes a Schrödinger-type equation of the minisuperspace variable $a$ (the scale factor) alone and can in principle be solved particularly in the absence of the cosmological constant.

Finally, our study of quantum wormholes in this work appears to demonstrate that, after all, the EYM theory is a simple yet fruitful system which serves as an arena in which we can envisage quite a few exciting aspects of quantum gravitational phenomena.

\section{Acknowledgements}

This work was supported in part by Korea Research Foundation and by Basic Science Research Institute (BSRI-97-2427) at Ewha Women's Univ. 


\section{References}

[1] E. Baum, Phys. Lett. B133, 185 (1983) ; S. W. Hawking, Phys. Lett. B134, 403 (1984).

[2] S. Giddings and A. Strominger, Nucl. Phys. B307, 854 (1988).

[3] S. Coleman, Nucl. Phys. B307, 864 (1988) ; Nucl. Phys. B310, 643 (1988) ; I. Klebanov, L. Susskind, and T. Banks, Nucl. Phys. B317, 665 (1989).

[4] S. Giddings and A. Strominger, Nucl. Phys. B306, 890 (1988) ; S. -J. Rey, Nucl. Phys. B319, 765 (1989).

[5] A. Hosoya and W. Ogura, Phys. Lett. B22, 117 (1989) ; A. K. Gupta et al., Nucl. Phys. B333, 195 (1990) ; S. -J. Rey, Nucl. Phys. B336, 146 (1990).

[6] K. Lee, Phys. Pev. Lett. 61, 263 (1988).

[7] B. Grinstein and M. B. Wise, Phys. Lett. B212, 407 (1988) ; B. Grinstein, Nucl. Phys. B321, 439 (1989).

[8] W. Fischler and L. Susskind, Phys. Lett. B217, 48 (1989).

[9] G. W. Gibbons, S. W. Hawking, and M. J. Perry, Nucl. Phys. B138, 141 (1978).

[10] S. W. Hawking and D. N. Page, Phys. Rev. D42, 2655 (1990).

[11] C. W. Misner, K. S. Thorne, and J. A. Wheeler, Gravitation (W. H. Freeman, San Francisco, 1973).

[12] J. B. Hartle and S. W. Hawking, Phys. Rev. D28, 2960 (1983) ; S. W. Hawking, Nucl. Phys. B239, 257 (1984). 
[13] A. Vilenkin, Phys. Lett. B117, 25 (1982) ; Phys. Rev. D30, 509 (1984) ; Nucl. Phys. B252, 141 (1985) ; Phys. Rev. D33, 3560 (1986) ; Phys. Rev. D37, 888 (1988). 


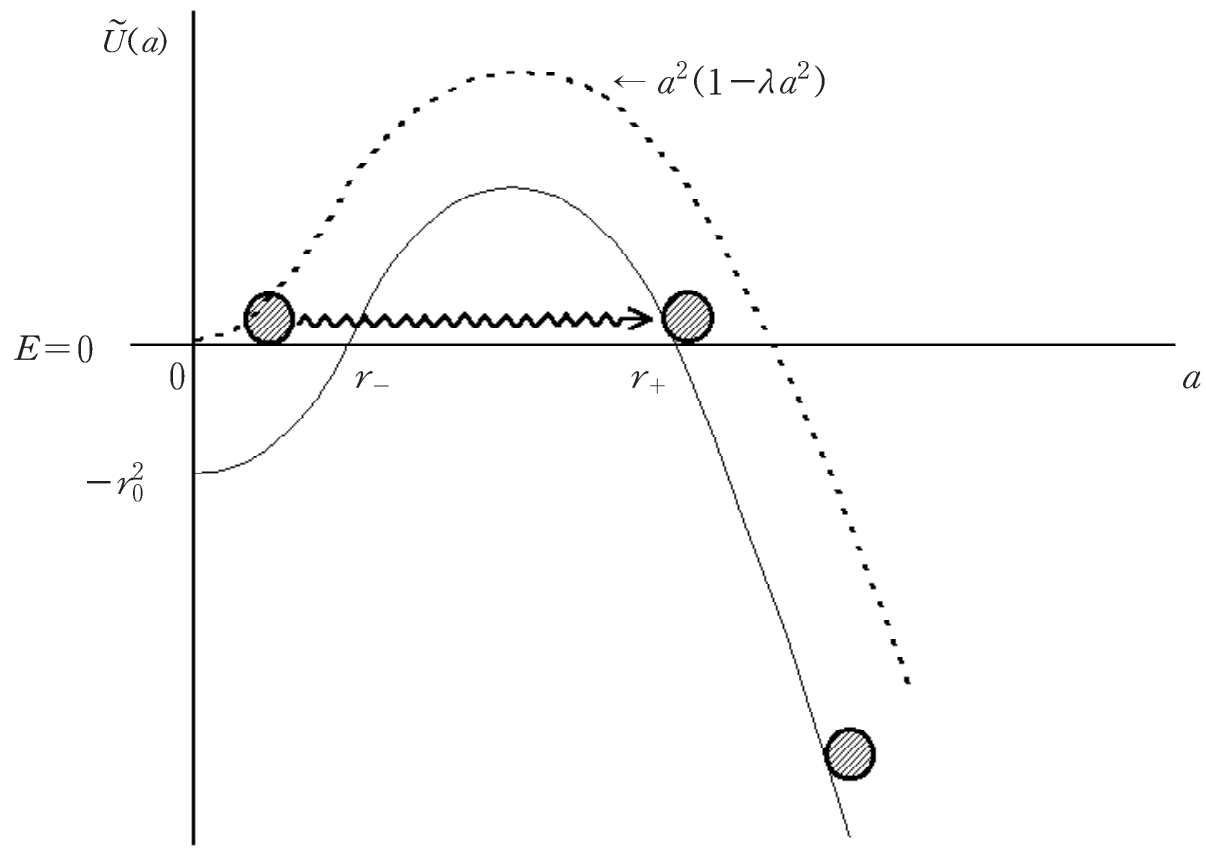

Fig.1 The plot of the potential energy as it appears in the WD equation in the presence of the cosmological constant.

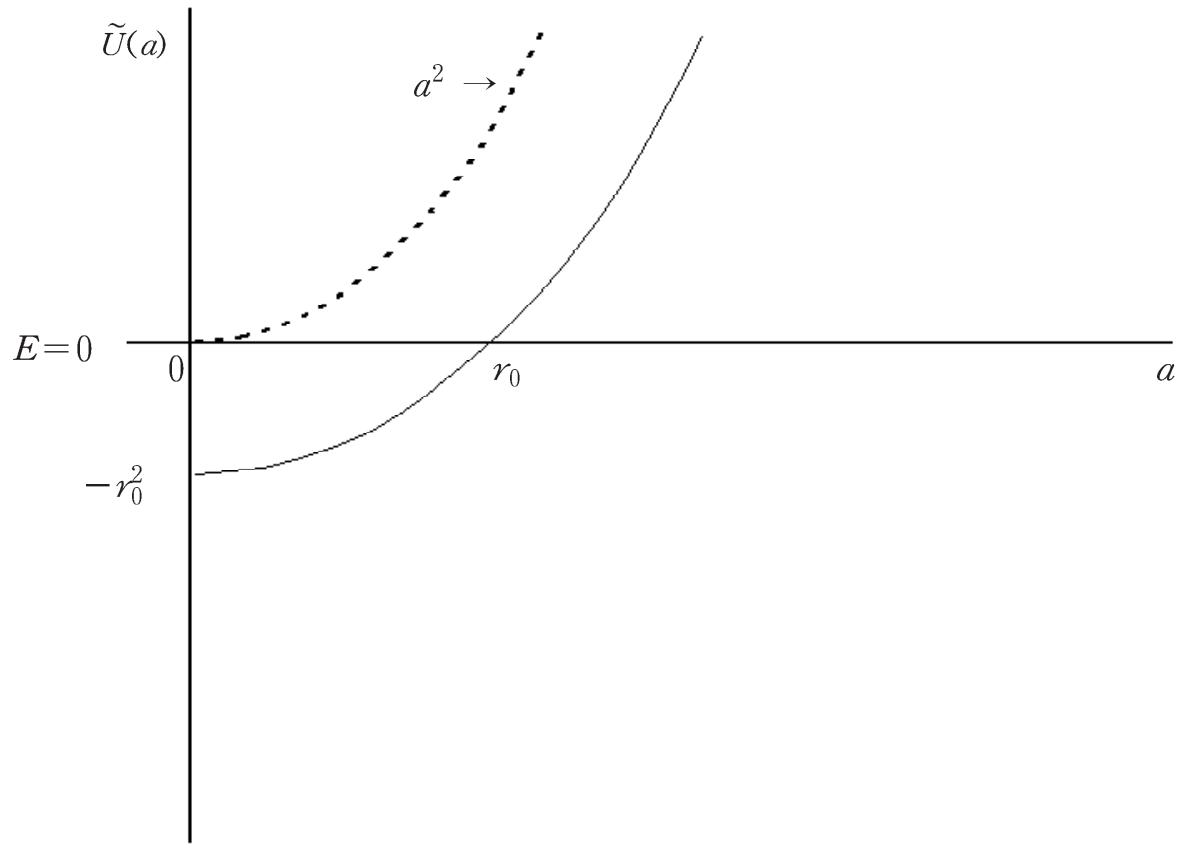

Fig.2 The plot of the potential energy as it appears in the WD equation in the absence of the cosmological constant. 


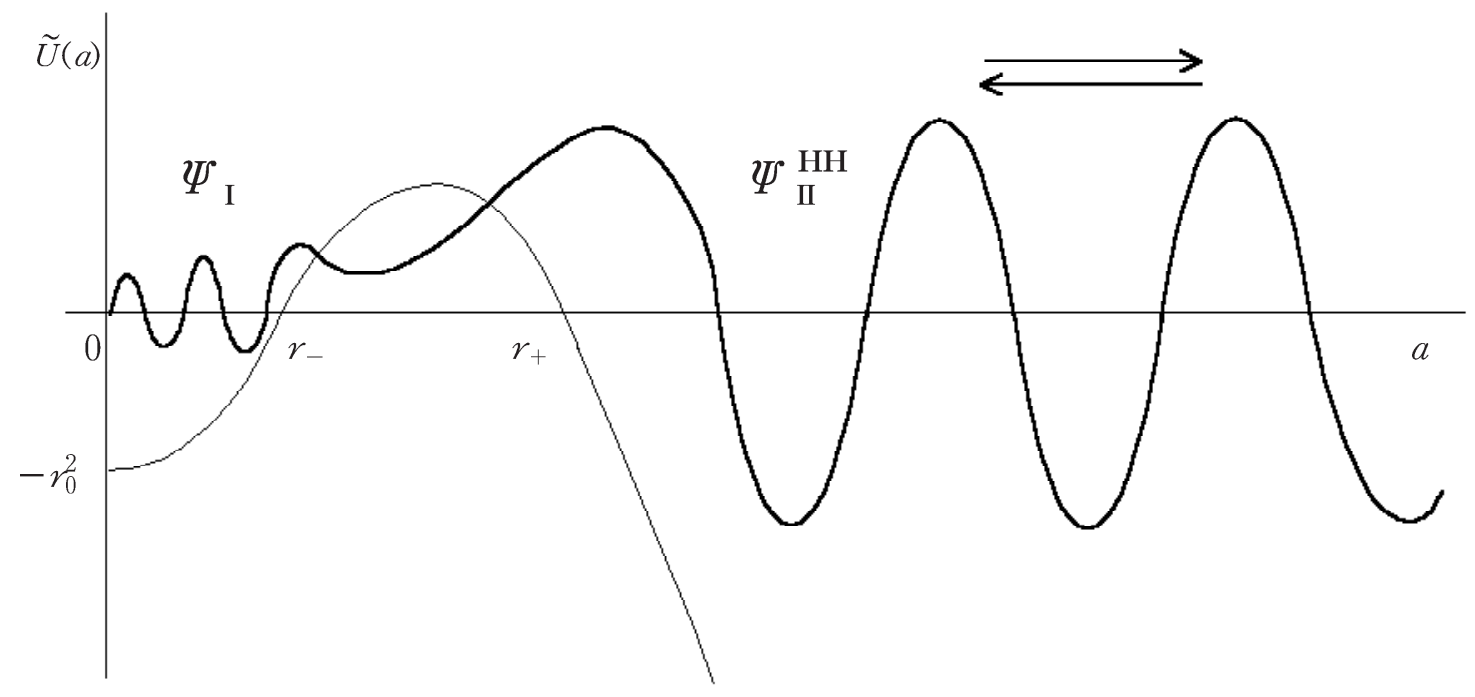

Fig.3 A regular solution to the WD equation in the presence of the cosmological constant employing $\mathrm{HH}^{\prime}$ s no-boundary proposal.

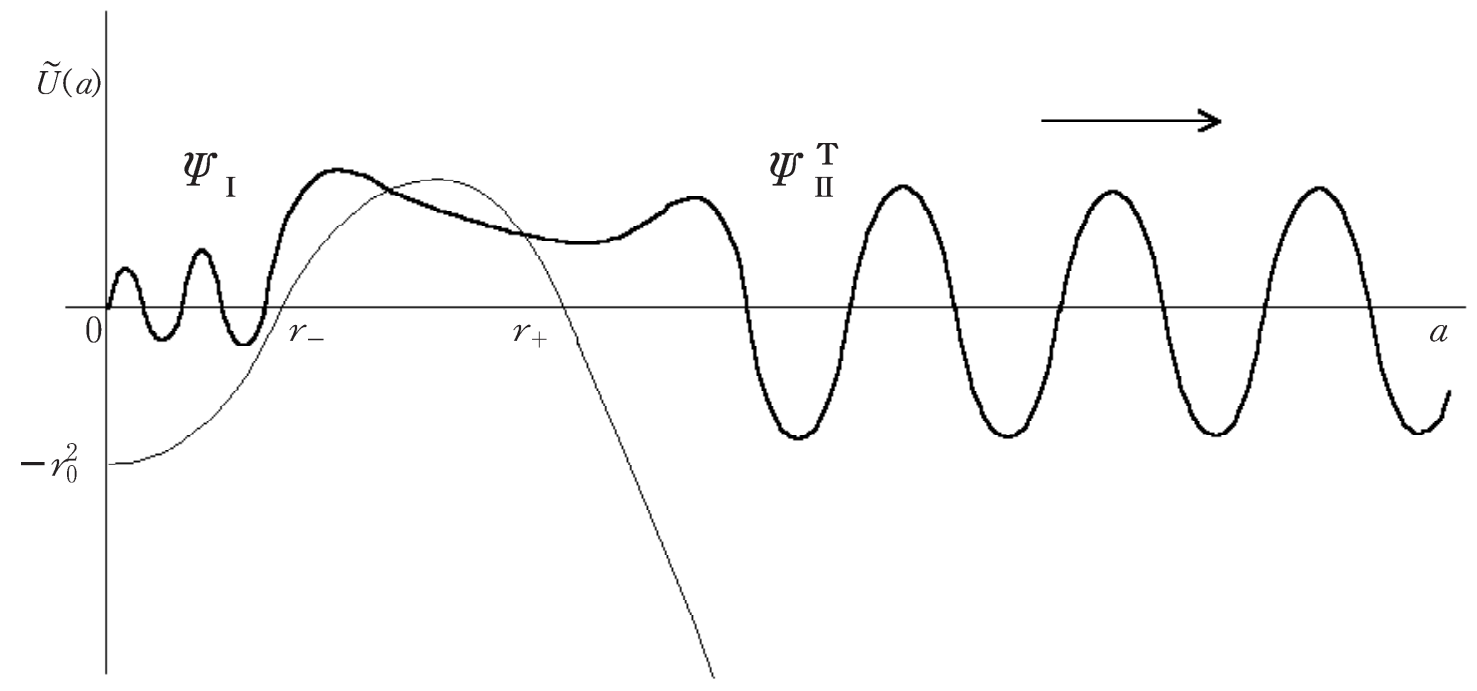

Fig.4 A regular solution to the WD equation in the presence of the cosmological constant employing Vilenkin's tunnelling boundary condition. 